\title{
Macular pigment optical density: repeatability, intereye correlation, and effect of ocular dominance
}

This article was published in the following Dove Press journal:

Clinical Ophthalmology

29 August 2016

Number of times this article has been viewed

\section{Pinakin Gunvant Davey \\ Silverio D Alvarez \\ Jessica $Y$ Lee \\ College of Optometry, Western University of Health Sciences, \\ Pomona, CA, USA}

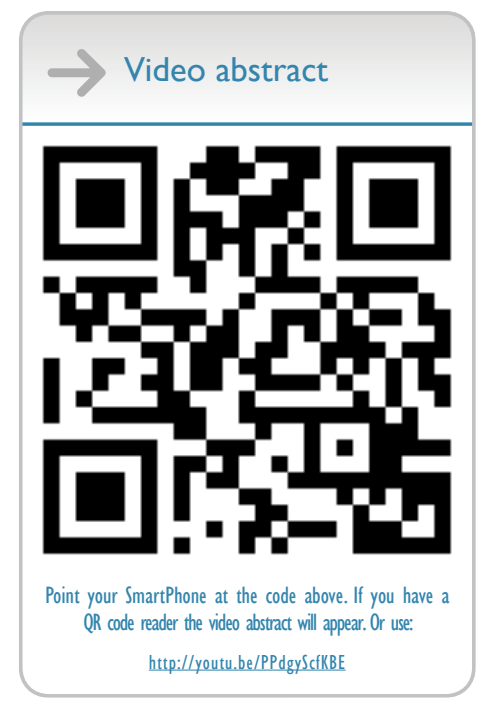

Correspondence: Pinakin Gunvant Davey College of Optometry, Western University of Health Sciences, 309 E Second Street, Pomona, CA 91766, USA Tel +l 9094698473

Email contact@pinakin-gunvant.com
Purpose: To evaluate short-term repeatability, intereye correlation, and effect of ocular dominance on macular pigment optical density (MPOD) measurements obtained using the QuantifEye Heterochromatic Flicker Photometer.

Patients and methods: A total of 72 study participants were enrolled in this prospective, cross-sectional study. Participants underwent a comprehensive ocular evaluation, including visual acuity, evaluation of ocular dominance, slit lamp examination, intraocular pressure measurement, and optic nerve head and macula analysis using optical coherence tomography and fundus photography. All study participants after initial training underwent MPOD measurement twice in both eyes in a randomized sequence. The repeatability was tested using Altman and Bland plots for first measurements with the second measurements for right eye and left eye and additionally by grouping eyes as a function of ocular dominance. The Pearson correlation coefficient was performed to assess the intereye correlation of MPOD values.

Results: The mean age of study participants was 35.5 years (range $22-68$ years). The mean MPOD measurements for OD (right eye) and OS (left eye) were 0.47 and 0.48 , respectively, which followed a normal distribution (Shapiro-Wilk test, $P=0.6$ and 0.2 ). The $95 \%$ limits of agreement of Altman and Bland plots for the first and second measurements were -0.12 to +0.11 and -0.13 to +0.12 for OD and OS, respectively. The correlation coefficient of mean MPOD measurements of OD and OS was $r$ statistic $=0.94$ (Pearson correlation coefficient $P<0.0001 ; r^{2} 0.89$ ). The 95\% limits of agreement of Altman and Bland plots when evaluated by laterality of eye or by ocular dominance were narrow, with limits of agreement ranging from -0.13 to +0.12 .

Conclusion: The MPOD measurements obtained using the QuantifEye show good short-term repeatability. There is excellent intereye correlation, indicating that the MPOD values of one eye data can predict the fellow eye value with $89 \%$ accuracy. The ocular dominance had no bearing on the outcome of this psychophysical test in ocular healthy eyes.

Keywords: macular pigment optical density, heterochromatic flicker photometry, age-related macular degeneration, ocular dominance, QuantifEye, repeatability, clinical protocol

\section{Introduction}

Age-related macular degeneration (AMD) is a leading cause of blindness in developed countries worldwide. ${ }^{1-7}$ AMD is broadly classified into two categories, exudative AMD and nonexudative AMD ${ }^{8}$ Estimates indicate that in the US there were 2 million patients with late AMD and 8 million patients with intermediate AMD in 2004, and it is predicted that this number will double in the year $2024 .{ }^{9}$ There are numerous breakthroughs in the understanding of pathogenesis of wet AMD, and treatments have been developed in the form of antivascular endothelial growth factors. ${ }^{8}$ However, the 
understanding of the disease pathophysiology of the more common variety of AMD, the nonexudative AMD, is still lacking $^{8}$ and the literature is not consistent on the efficacy of treatment. ${ }^{10-12}$

The Age-Related Eye Study-2 (AREDS-2) ${ }^{10}$ investigated the role of nutritional supplements in the progression of AMD; they particularly investigated the role of carotenoids, lutein, and zeaxanthin along with omega-3 in the prevention of progression in AMD. ${ }^{10}$ The AREDS-2 investigators concluded that taking oral supplements of carotenoids such as lutein and zeaxanthin may have beneficial effect in preventing progression to the late-stage AMD. ${ }^{10}$ Various studies have shown that oral supplementation of carotenoids can increase the measured level of macular pigment optical density (MPOD) to a variable degree, ${ }^{13-20}$ and thus MPOD measurements can be one of the ways to evaluate the treatment efficacy of oral supplementation of carotenoids. MPOD can be measured clinically using various objective methods and methods requiring subjective response. ${ }^{21-24}$ The QuantifEye (Zeavision, St Louis, MO, USA) is one of the devices that can estimate MPOD clinically. ${ }^{25-29}$ Studies have utilized the QuantifEye for measurement of MPOD repeatability, ${ }^{25-29}$ however, data on repeatability and on standardized technique that can be utilized in clinics to measure MPOD are limited.

We sought to evaluate the QuantifEye in measuring MPOD in a group of ocular healthy individuals to get an understanding of the variability of measurement of MPOD due to human and ocular factors, with the idea of establishing protocols that can aid future studies. We investigated repeatability of measurements, intereye variability, and the effect of ocular dominance on MPOD measurements.

\section{Patients and methods}

The study was approved by the institutional review board at Western University of Health Sciences, Pomona, California, USA, and conducted in accordance with the tenets of the Declaration of Helsinki. A group of 72 individuals were evaluated for the study and all participants provided signed informed consent for the study. All participants were at least 18 years of age and were deemed ocular healthy by a dilated comprehensive examination in the last 1 year. Additionally, all individuals had a study-specific eye examination that included visual acuity measurement using the logMAR charts, ocular examination using the slit lamp biomicroscope, tonometry using the Ocular Response Analyzer, and optic disk and macula examination using the optical coherence tomography and fundus photography of the posterior pole. Participants with diabetic retinopathy, macular degeneration, macular edema, prior history of retinal detachment or vitreoretinal surgeries, or other congenital retinal pathologies or significant media opacity that could influence the outcome of the study were not included. The study participants in the immediate postoperative follow-up period (3 months) of cataract surgery or invasive ocular surgery were also not included. The presence of retinal disease or glaucoma was evaluated objectively using optical coherence tomography scans and fundus photographs during the study visit. All participants had a logMAR visual acuity of $+0.2(20 / 32)$ or better. The mean visual acuity of the study population was 0.003 (standard deviation [SD] 0.08) and 0.002 (SD 0.09) for the right and left eyes, respectively. The mean logMAR visual acuity was not significantly different on the basis of laterality of the eye and ocular dominance ( $P$-value paired samples $t$-test $P=0.96$ and $P=0.19$, respectively).

\section{Identification of the dominant eye}

The Miles technique ${ }^{30}$ of identifying ocular dominance was utilized in the study. Briefly, all participants were asked to look at a small target at a distance of $10 \mathrm{ft}$. Participants were instructed to view the target with both their eyes through a small aperture made by their hands at arm's length. The eyes were closed one at a time and the participants were asked to report which eye maintained the fixation and was seeing the distant target. The eye that maintained the fixation was deemed as the dominant eye.

\section{MPOD measurements}

MPOD measurements were obtained using the QuantifEye by trained observers who were part of the study group. The QuantifEye is a heterochromatic flicker photometer (HFP) that can measure MPOD in vivo. The equipment details about heterochromatic flicker photometry can be found elsewhere. ${ }^{24,25,31}$ Briefly, the mode of action of heterochromatic flicker photometry is based on the macular pigment's absorption spectrum at the retina, specifically the macula and fovea. ${ }^{24,25,31}$ The heterochromatic flicker photometry determines the MPOD by displaying two light stimuli of different wavelengths, which the patient perceives as a flicker. ${ }^{24,25,31}$ The light stimuli alternate between a blue light of short wavelength, which is maximally absorbed by the macular pigments, and a greenyellow light of longer wavelength, which is not absorbed by the pigments. ${ }^{24,25,31}$

First, the flickering light is targeted to the fovea; upon perceiving the stimulus the subject will respond that "the target is flickering". The first five targets determine the 
threshold. The testing is continued and the machine alters the radiance of blue light versus the green-yellow light until the patient perceives no flicker and is recorded as the lowest point on the graph given in Figure 1. The machine continues to increase the blue radiance until a flicker is perceived three times consecutively. The machine has a built-in correction factor to account for the age-related yellowing of the lens and a final MPOD measurement is obtained. If the individual being examined had an intraocular lens implant, the age of participant is recorded as 21 years where the machines' correction factor for the yellowing of the lens is zero.
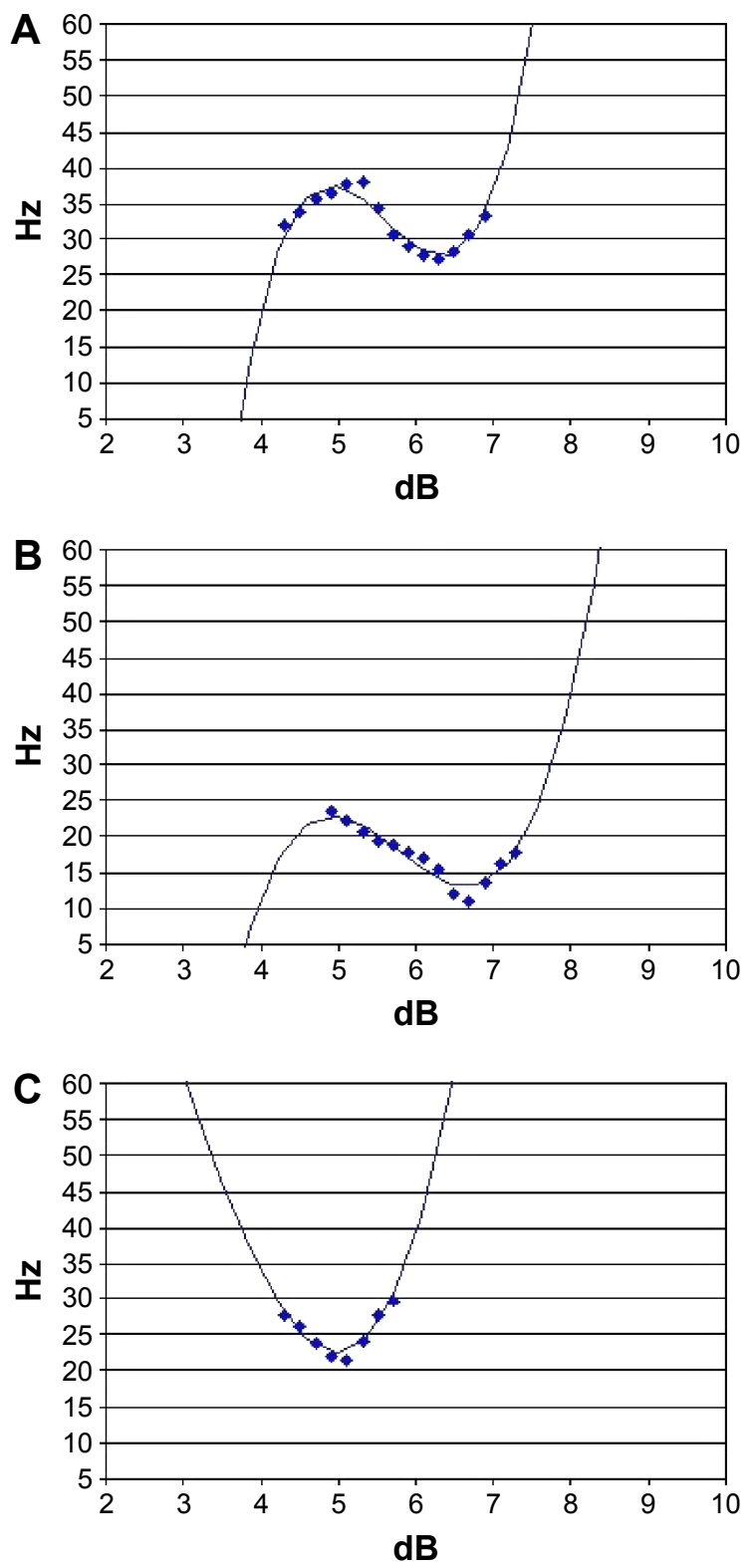

All participants had a brief tutorial explaining the procedure of testing and were shown a picture of what target will be visible during testing. The eye undergoing testing first was randomly selected and then the fellow eye was examined. Then the procedure was repeated in both eyes. Participants were given rest when desired between tests. Each eye testing takes $\sim 2$ minutes to perform the procedure. The device outputs include a graph and a value of the MPOD. The graphs (Figure 1) are evaluated for reliability using a set criterion in evaluating the graphs. For a result to be deemed acceptable the graph should have the following criteria: 1) there
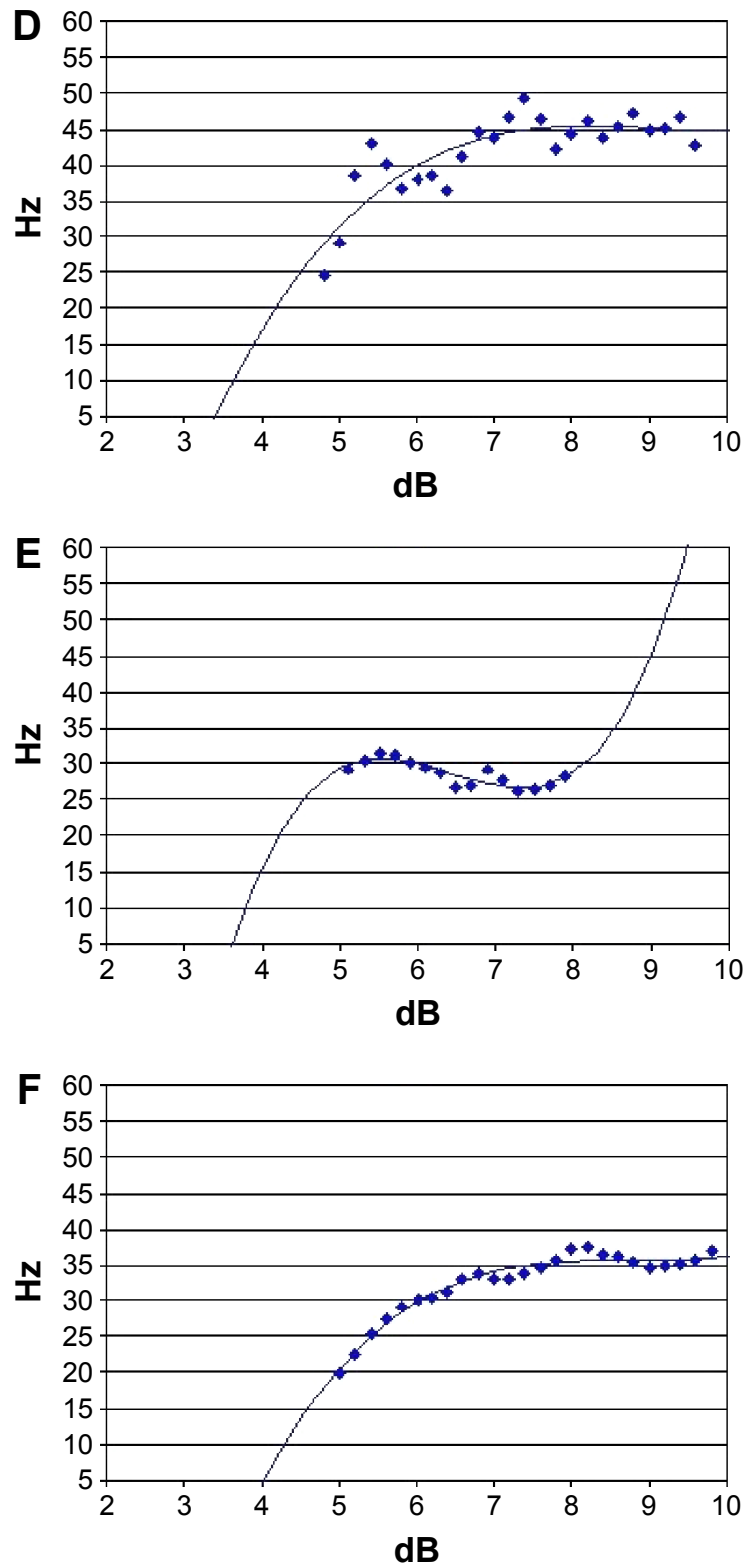

Figure I MPOD graphs showing acceptable and unacceptable examples.

Notes: (A-C) Left panels show S-shaped, V-shaped, or U-shaped MPOD output graphs, respectively, that have a lowest point and were considered acceptable. Right panels (D-F) show MPOD graphs that were considered to have data with excessive variability, no downward trend and/or no definite low point and was considered unacceptable. Abbreviation: MPOD, macular pigment optical density. 
has to be a downward slope of data points, 2) a distinct lowest point, and 3) three points in upward direction from the lowest point.

\section{Statistical analysis}

The difference in number of male and female participants was tested using the binomial test with hypothesized proportions 0.5 . The normality of distribution was tested using the Shapiro-Wilk test. The repeatability of the first and second set of MPOD measurements obtained using the right and left eyes and the dominant and nondominant eyes was assessed using the Altman and Bland plots. The paired samples $t$-test was utilized to evaluate the differences between first and second measurements of MPOD obtained from right eye and left eye and dominant eye and the nondominant eye. The coefficient of repeatability was also calculated. Pearson correlation coefficient was utilized to evaluate the correlation between mean MPOD obtained between the right and left eyes. One-way analysis of variance (ANOVA) was utilized to evaluate the difference in mean MPOD as a function of sex. The statistical analysis was performed using Analyze-it for Microsoft Excel v2.26.

\section{Results}

The mean age of the study participants was 35.5 (SD 12.4, range 22-68 years). The study participants consisted of approximately equal number of males (29) and females (43); the difference in the number of males and females was not statistically significant (binomial test proportion $0.40, P=0.12$ ).

Table 1 provides the mean MPOD measurements of the study population grouped by laterality, ocular dominance, sex, and coefficient of repeatability. The mean MPOD measurements was not significantly different when participants were grouped by ocular dominance or laterality (paired samples $t$-test, $t$ statistic $=0.42, P=0.68$ and $t$ statistic $=-1.08$, $P=0.28$, respectively). The mean MPOD measurements in the dominant eye were 0.45 (SD 0.19) and 0.44 (SD 0.14) for males and females, respectively, and not significantly different (one-way ANOVA $F=0.05, P=0.8$ ).

\section{Assessment of repeatability of MPOD measurements}

Figure 1 shows the Altman and Bland plots of the first and second measurements obtained on the right eye and the left eyes, respectively. There is no significant systematic bias between the first and second measurements of the right and left eyes, respectively, with the mean difference between the first and second measurements being close to zero (bias $=-0.002$ and -0.001 , respectively, for the right and left eyes). The difference in the first and second measurements obtained was not significantly different for the data obtained on the right and left eyes (paired samples $t$-test, $t$ statistic $-0.26, P=0.80$ and $t$ statistic $-0.09, P=0.93$ for the right and left eyes, respectively).

The limits of agreement were narrow, indicating good repeatability of measurements (Figure $2 \mathrm{~A}$ and $\mathrm{B}$ for values) when evaluating measurements grouped by laterality. The coefficient of repeatability was small and in the range of 0.11 and 0.12 for the dominant and nondominant eyes, respectively.

\section{Assessment of intereye correlation of MPOD measurements}

Pearson correlation coefficient of mean MPOD values of the right and left eyes shows excellent correlation with $r$ statistic 0.94 ( $t$ statistic $=22.55, P=<0.0001)$. The equation allows us to calculate left eye MPOD values from right eye values with an accuracy of $89 \%$.

Left eye MPOD $=1.0272 \times$ right eye MPOD -0.0047

where MPOD is the macular pigment optical density measurement obtained using the QuantifEye device.

\section{Assessment of ocular dominance on MPOD measurements}

The repeatability of the MPOD is not influenced if eyes are grouped by ocular dominance. Figure $3 \mathrm{~A}$ and $\mathrm{B}$ shows the Altman and Bland plots of first and second measurements

Table I Values of macular pigment optical density as measured using the QuantifEye Heterochromatic Flicker Photometer

\begin{tabular}{|c|c|c|c|c|c|}
\hline \multirow[t]{2}{*}{ Eye tested } & \multicolumn{5}{|c|}{ Macular pigment optical density } \\
\hline & Mean & SD & Range & Limits of agreement & Coefficient of repeatability \\
\hline Right eye & 0.43 & 0.15 & $0.1-0.8 \mid$ & -0.11 to +0.11 & 0.11 \\
\hline Left eye & 0.44 & 0.17 & $0.1-0.91$ & -0.13 to +0.12 & 0.12 \\
\hline Dominant eye & 0.44 & 0.17 & $0.1-0.91$ & -0.12 to +0.11 & 0.12 \\
\hline Nondominant eye & 0.43 & 0.16 & $0 .|-0.8|$ & -0.13 to +0.12 & 0.13 \\
\hline
\end{tabular}

Abbreviation: SD, standard deviation. 

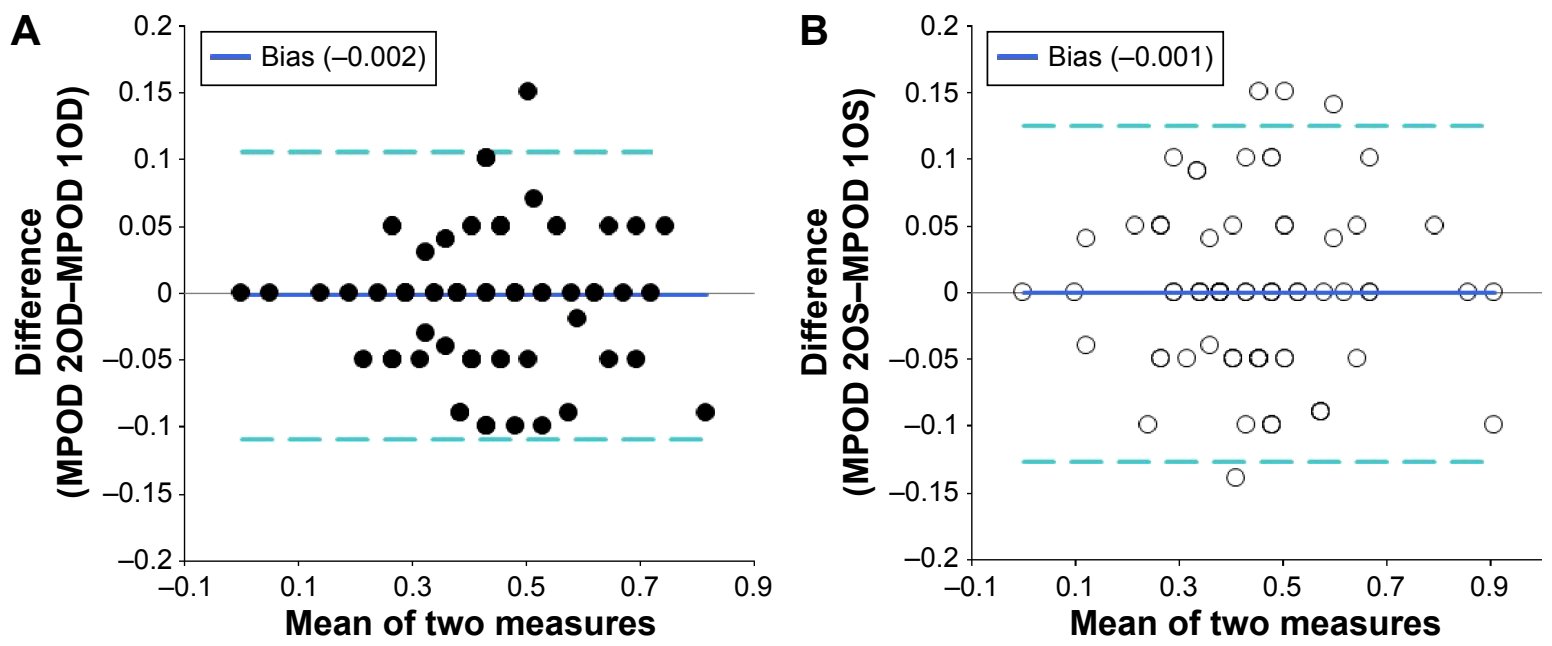

Figure 2 Repeatability of MPOD measurements.

Notes: (A) and (B) shows Altman and Bland plots with repeat MPOD measurements obtained using the right and left eyes, respectively. The data shows narrow limits of agreement and no significant bias.

Abbreviation: MPOD, macular pigment optical density.

obtained on the dominant and nondominant eyes, respectively. There is no significant systematic bias between the first and second measurements when grouped by ocular dominance with the mean difference between the first and second measurements being close to zero (bias $=-0.002$ and -0.001 , respectively, for the dominant and nondominant eyes, respectively). The difference in the first and second measurements obtained was not significantly different for the data grouped by dominance (paired samples $t$-test, $t$ statistic $-0.26, P=0.80$ and $t$ statistic $-0.09, P=0.93$ for the dominant and nondominant eyes, respectively). Furthermore, the difference in the first and second measurements was not significantly different between the dominant and nondominant eyes (paired $t$-test, $t$ statistic $=0.15, P=0.88$ ).

The limits of agreement were narrow, indicating good repeatability of measurements (Table 1 for values) when evaluating measurements grouped by dominance. The mean MPOD measured in both the dominant and nondominant eyes was not significantly different in males and females (one-way ANOVA $F$ statistic $=0.05, P=0.82$ and $F$ statistic $=0.01, P=0.94)$. The mean MPOD measurements for the dominant and nondominant eyes were 0.447 and 0.444 , respectively, which were not significantly different (paired samples $t$-test $=0.42, P=0.68$ ).
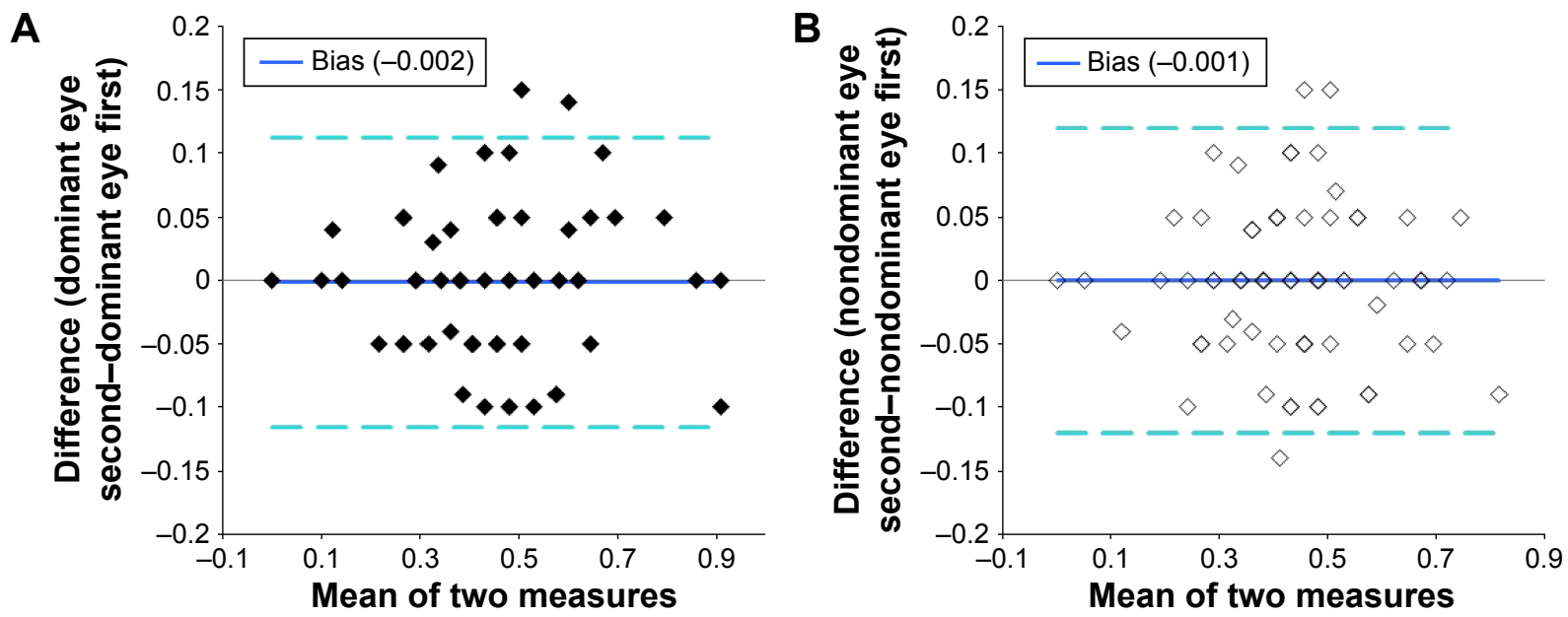

Figure 3 Repeatability of measures in dominant and nondominant eye.

Notes: (A) and (B) shows Altman and Bland plots with repeat MPOD measurements as grouped by ocular dominance. The data shows narrow limits of agreement and no significant bias.

Abbreviation: MPOD, macular pigment optical density. 


\section{Discussion}

The results of this study show that by utilizing standardized techniques and minimal training, novice individuals can perform testing with the HFP (QuantifEye) and repeatable measurements can be obtained. This study also looked at various factors that could be important to consider when examining patients clinically. This study also establishes protocols for future studies.

Prior studies have looked at repeatability of MPOD measurements obtained using the QuantifEye HFP. The literature was not consistent with some studies finding the repeatability not acceptable, ${ }^{25,28}$ whereas some other finding the repeatability acceptable. ${ }^{26,29}$ All these studies ${ }^{25,28}$ have smaller sample sizes ranging from 23 to 40 individuals, whereas the present study had a relatively larger sample size. The possible explanation of the difference in results lies in the quantity and quality of data collected and understanding of the tests by the individuals. Howells et $\mathrm{al}^{27}$ found that removing the suboptimal graphs allowed for improvement in coefficient of repeatability by 0.04 and intersession repeatability by 0.08 . The QuantifEye HFP is a psychophysical test and requires some degree of understanding of the tests. Thus, an output of threshold that is graphed also needs to be interpreted by trained observers. In the present study, we found that standardized minimal explanation to individuals performing the MPOD testing and quality check of the MPOD graphs obtained, yields reproducible MPOD results with limits of agreement being narrow and the coefficient of repeatability being $0.11-0.12$. The coefficient of repeatability is clinically useful information when augmenting supplements for improving MPOD in individuals at risk of AMD. An increase in MPOD values $>0.12$ can be considered to be significant.

The results of the present study indicate that the measurements obtained in both the right and left eyes of an individual are repeatable. There was no significant difference in measurements obtained on both right and left eyes. This is consistent with prior studies. ${ }^{32-34}$ Furthermore, the MPOD values obtained from one eye can predict the values of the fellow eye with $89 \%$ accuracy. The MPOD values between eyes are not significantly different because the carotenoids, lutein and zeaxanthin, are obtained from oral route and perhaps deposited equally in both eyes. The clinical relevance of the results overall indicates that clinically in ocular healthy individuals it will be sufficient to obtain results in one eye as an indicator of an individual's MPOD measurements.

The present study evaluated the issue of MPOD in ocular dominant eye versus nondominant eye in detail. It was probable that the individuals may be better at performing a psychophysical test with their ocular dominant eye compared with the nondominant eye. It was also possible that the MPOD levels could be greater in the dominant eye and it could in part be responsible for slightly greater visual function and may explain ocular dominance. Although the mean MPOD of the dominant eye was marginally higher than the mean MPOD of the nondominant eye $(0.447$ vs 0.444 , respectively), the difference was not statistically significant and unlikely of any clinical significance. The difference in first set of MPOD measurements and second set of measurements, repeatability, and the mean MPOD values was not related to ocular dominance. It can be safely concluded that the MPOD values can be obtained from either eye, although clinically the patients may feel comfortable using the dominant eye over nondominant eye when performing the test.

The known risk factors of the nonexudative AMD include age, smoking, sunlight exposure, race, and low macular pigment. ${ }^{8}$ Of the known risk factors, one of the modifiable risk factors is the level of MPOD in the retina. It is postulated that increasing the MPOD allows for the absorption of blue light and quenching of the free radicals in the retina, which may harm the retina. The AREDS- $2^{10}$ trial showed that oral supplementation of carotenoids may aid and could be beneficial in prevention of AMD progression to advanced stages. Unfortunately, the trial did not measure MPOD. The baseline level of MPOD and change in MPOD with oral supplementation may aid in clinical decision making and adjusting the dose of oral supplementation to achieve desired effect and not experience unnecessary side effects due to oral supplementation of these carotenoids. Therefore, it is clinically important to accurately measure MPOD. The current study shows that the device QuantifEye can yield repeatable MPOD measurements with low variability. The present study also outlines an examination protocol that can be utilized in future studies that evaluate MPOD.

\section{Acknowledgments}

The authors would like to thank Tom Thamsopit, Roshini Shah, Pratik Vyas, Bridgett Santos, and Samantha Zaczyk for their assistance in data collection. The authors would like to acknowledge intramural grant support from Western University of Health Sciences and unrestricted grant and equipment support from Zeavision LLC to investigate MPOD. The authors would also like to thank Dennis Geirhart, PhD, for useful discussions. The abstract of this paper was presented at the annual meeting of American Academy of Optometry Seattle, USA, 2013, as a poster 
presentation with interim findings. The poster's abstract was published in "Poster Abstracts" online program number 135209: http://www.aaopt.org/macular-pigment-opticaldensity-repeatability-inter-eye-correlation-and-effect-oculardominance.

\section{Disclosure}

The authors report no conflicts of interest in this work.

\section{References}

1. Friedman DS, O'Colmain BJ, Munoz B, et al; Eye Diseases Prevalence Research Group. Prevalence of age-related macular degeneration in the United States. Arch Ophthalmol. 2004;122(4):564-572.

2. Furtado JM, Lansingh VC, Carter MJ, et al. Causes of blindness and visual impairment in Latin America. Surv Ophthalmol. 2012;57(2): 149-177.

3. Hawkins BS, Bird A, Klein R, West SK. Epidemiology of age-related macular degeneration. Mol Vis. 1999;5:26.

4. Owen CG, Jarrar Z, Wormald R, Cook DG, Fletcher AE, Rudnicka AR. The estimated prevalence and incidence of late stage age related macular degeneration in the UK. Br J Ophthalmol. 2012;96(5):752-756.

5. Rudnicka AR, Jarrar Z, Wormald R, Cook DG, Fletcher A, Owen CG. Age and gender variations in age-related macular degeneration prevalence in populations of European ancestry: a meta-analysis. Ophthalmology. 2012;119(3):571-580.

6. Spanish Eyes Epidemiological Study Group. Prevalence of agerelated macular degeneration in Spain. Br J Ophthalmol. 2011;95(7): 931-936.

7. Vingerling JR, Klaver CC, Hofman A, de Jong PT. Epidemiology of age-related maculopathy. Epidemiol Rev. 1995;17(2):347-360.

8. Ambati J, Fowler BJ. Mechanisms of age-related macular degeneration. Neuron. 2012;75(1):26-39.

9. Congdon N, O'Colmain B, Klaver CC, et al. Causes and prevalence of visual impairment among adults in the United States. Arch Ophthalmol. 2004;122(4):477-485.

10. Age-Related Eye Disease Study 2 Research Group. Lutein + zeaxanthin and omega-3 fatty acids for age-related macular degeneration: the Age-Related Eye Disease Study 2 (AREDS2) randomized clinical trial JAMA. 2013;309(19):2005-2015.

11. Age-Related Eye Disease Study Research Group. A randomized, placebo-controlled, clinical trial of high-dose supplementation with vitamins $\mathrm{C}$ and $\mathrm{E}$, beta carotene, and zinc for age-related macular degeneration and vision loss: AREDS report no. 8. Arch Ophthalmol. 2001; 119(10):1417-1436.

12. Aronow ME, Chew EY. Age-related eye disease study 2: perspectives, recommendations, and unanswered questions. Curr Opin Ophthalmol. 2014;25(3):186-190

13. Akuffo KO, Beatty S, Stack J, et al. Central retinal enrichment supplementation trials (CREST): design and methodology of the CREST randomized controlled trials. Ophthalmic Epidemiol. 2014;21(2): $111-123$

14. Berendschot TT, Goldbohm RA, Klopping WA, van de Kraats J, van Norel J, van Norren D. Influence of lutein supplementation on macular pigment, assessed with two objective techniques. Invest $\mathrm{Oph}$ thalmol Vis Sci. 2000;41(11):3322-3326.

15. Khachik F, de Moura FF, Chew EY, et al. The effect of lutein and zeaxanthin supplementation on metabolites of these carotenoids in the serum of persons aged 60 or older. Invest Ophthalmol Vis Sci. 2006; 47(12):5234-5242.

16. Koh HH, Murray IJ, Nolan D, Carden D, Feather J, Beatty S. Plasma and macular responses to lutein supplement in subjects with and without agerelated maculopathy: a pilot study. Exp Eye Res. 2004;79(1):21-27.
17. Ma L, Yan SF, Huang YM, et al. Effect of lutein and zeaxanthin on macular pigment and visual function in patients with early age-related macular degeneration. Ophthalmology. 2012;119(11):2290-2297.

18. Richer S, Devenport J, Lang JC. LAST II: differential temporal responses of macular pigment optical density in patients with atrophic age-related macular degeneration to dietary supplementation with xanthophylls. Optometry. 2007;78(5):213-219.

19. Trieschmann M, Beatty S, Nolan JM, et al. Changes in macular pigment optical density and serum concentrations of its constituent carotenoids following supplemental lutein and zeaxanthin: the LUNA study. Exp Eye Res. 2007;84(4):718-728.

20. Weigert G, Kaya S, Pemp B, et al. Effects of lutein supplementation on macular pigment optical density and visual acuity in patients with agerelated macular degeneration. Invest Ophthalmol Vis Sci. 2011;52(11): 8174-8178.

21. Berendschot TT, van Norren D. Objective determination of the macular pigment optical density using fundus reflectance spectroscopy. Arch Biochem Biophys. 2004;430(2):149-155.

22. Bernstein PS, Yoshida MD, Katz NB, McClane RW, Gellermann W. Raman detection of macular carotenoid pigments in intact human retina. Invest Ophthalmol Vis Sci. 1998;39(11):2003-2011.

23. Delori FC, Goger DG, Hammond BR, Snodderly DM, Burns SA. Macular pigment density measured by autofluorescence spectrometry: comparison with reflectometry and heterochromatic flicker photometry. J Opt Soc Am A Opt Image Sci Vis. 2001;18(6):1212-1230.

24. Howells O, Eperjesi F, Bartlett H. Measuring macular pigment optical density in vivo: a review of techniques. Graefes Arch Clin Exp Ophthalmol. 2011;249(3):315-347.

25. Bartlett H, Stainer L, Singh S, Eperjesi F, Howells O. Clinical evaluation of the MPS 9000 macular pigment screener. Br J Ophthalmol. 2010;94(6):753-756.

26. de Kinkelder R, van der Veen RL, Verbaak FD, Faber DJ, van Leeuwen TG, Berendschot TT. Macular pigment optical density measurements: evaluation of a device using heterochromatic flicker photometry. Eye (Lond). 2011;25(1):105-112.

27. Howells O, Eperjesi F, Bartlett H. Improving the repeatability of heterochromatic flicker photometry for measurement of macular pigment optical density. Graefes Arch Clin Exp Ophthalmol. 2013;251(3): 871-880.

28. Loughman J, Scanlon G, Nolan JM, O'Dwyer V, Beatty S. An evaluation of a novel instrument for measuring macular pigment optical density: the MPS 9000. Acta Ophthalmol. 2012;90(2):e90-e97.

29. van der Veen RL, Berendschot TT, Hendrikse F, Carden D, Makridaki M, Murray IJ. A new desktop instrument for measuring macular pigment optical density based on a novel technique for setting flicker thresholds. Ophthalmic Physiol Opt. 2009;29(2):127-137.

30. Mapp AP, Ono H, Barbeito R. What does the dominant eye dominate? A brief and somewhat contentious review. Percept Psychophys. 2003; 65(2):310-317.

31. Leung IY. Macular pigment: new clinical methods of detection and the role of carotenoids in age-related macular degeneration. Optometry. 2008;79(5):266-272.

32. Beatty S, Murray IJ, Henson DB, Carden D, Koh H-H, Boulton ME. Macular pigment and risk for age-related macular degeneration in subjects from a Northern European population. Invest Ophthalmol Vis Sci. 2001;42(2):439-446.

33. Iannaccone A, Mura M, Gallaher KT, et al. Macular pigment optical density in the elderly: findings in a large biracial Midsouth population sample. Invest Ophthalmol Vis Sci. 2007;48(4):1458-1465.

34. Snodderly DM, Mares JA, Wooten BR, et al; CAREDS Macular Pigment Study Group. Macular pigment measurement by heterochromatic flicker photometry in older subjects: the carotenoids and age-related eye disease study. Invest Ophthalmol Vis Sci. 2004;45(2):531-538. 


\section{Publish your work in this journal}

Clinical Ophthalmology is an international, peer-reviewed journal covering all subspecialties within ophthalmology. Key topics include: Optometry; Visual science; Pharmacology and drug therapy in eye diseases; Basic Sciences; Primary and Secondary eye care; Patient Safety and Quality of Care Improvements. This journal is indexed on

Submit your manuscript here: http://www.dovepress.com/clinical-ophthalmology-journal
PubMed Central and CAS, and is the official journal of The Society of Clinical Ophthalmology (SCO). The manuscript management system is completely online and includes a very quick and fair peer-review system, which is all easy to use. Visit http://www.dovepress.com/ testimonials.php to read real quotes from published authors. 